\title{
Exploring piezoelectric properties of III-V nanowires using piezo-response force microscopy
}

\author{
Yonatan Calahorra ${ }^{1}$
}

Xin Guan ${ }^{2}$

Nripendra N. Halder ${ }^{3}$

Michael Smith ${ }^{1}$

Shimon Cohen ${ }^{3}$

Dan Ritter ${ }^{3}$

Jose Penuelas ${ }^{2}$

\author{
Sohini Kar-Narayan ${ }^{1}$ \\ ${ }^{1}$ Department of Materials Science and Metallurgy, University of Cambridge, CB3 \\ 0FS, Cambrdige, UK \\ ${ }^{2}$ Institut des Nanotechnologies de Lyon, Université de Lyon, UMR 5227 - CNRS, \\ Ecole Centrale de Lyon, 36 avenue Guy de Collongue, F-69134 Ecully Cedex, France \\ ${ }^{3}$ Department of Electrical Engineering, Technion - IIT, 32000, Haifa, Israel \\ E-mail: sk568@cam.ac.uk
}

\begin{abstract}
Semiconducting III-V materials exhibiting piezoelectric properties are much sought after due to their potential applications in piezotronic and piezophototronic devices. Nanowires of III-V semiconductors are particularly interesting in this respect due to the occurrence of the wurtzite (WZ) structure commonly associated with enhanced piezoelectric properties in these materials, as opposed to the zinc blende (ZB) structure that is typically observed in the bulk. However, direct measurements of the piezoelectric properties of III-V nanowires using piezo-response force microscopy (PFM) is challenging, and the analysis and interpretation of such measurements is far from trivial. Here we present detailed finite element simulations of single GaAs nanowires, with both WZ and ZB crystalline structure, scanned by an atomic force microscope tip in PFM mode, demonstrating the effect of the non-uniform electric field between the tip and nanowire, scan direction as well as nanowire orientation on the resulting PFM signal. We also report PFM data from single GaAs and InP nanowires with both ZB and WZ structure, grown by molecular beam epitaxy, based on a novel
\end{abstract}


non-destructive intermittent contact PFM mode. We explain our experimental data in the framework of our simulations, and for the first time, extract an experimental value for the axial piezoelectric coefficient of WZ InP, $d_{33}=0.7-1 \mathrm{pm} / \mathrm{V}$. The methods and analysis described here are particularly relevant for the investigation of piezoelectric properties in a wide range of semiconducting III-V nanowire systems.

PACS numbers: 00.00, 20.00, 42.10

Keywords: III-V nanowires, piezoelectricity, Wurtzite, PFM, QNM Submitted to:

Semicond. Sci. Technol. 


\section{Introduction}

The interest in semiconductor piezoelectricity at the nanoscale has grown tremendously during the past decade, with interest focused primarily on $\mathrm{ZnO},[1,2]$ through pioneering work from Z.L. Wang's group, and III-N (mostly GaN) materials [3]. In both cases, the wurtzite (WZ) crystal structure induces spontaneous polarization in the c-axis, and enhanced piezoelectric coefficients $[4,5]$. During this period, demonstrated applications varied from sensing $[2,6]$ to energy harvesting [1,7] and piezo-phototronics $[8,9]$. Simultaneously, the growth and properties of III-V (mostly non-nitride) nanowires (NWs) were extensively studied, mainly for electronic $[10,11]$ and opto-electronic $[12,13]$ applications.

Interestingly, III-V NWs may form in the WZ structure [14-16], rather than the zinc-blende (ZB) crystal structure which is prevalent in bulk form [17]. Although presently well-known and controlled $[15,18,19]$ to a substantial extent, this discovery was unexpected in the early days of III-V NW research [20]. Moreover, even though the WZ crystal structure is expected to induce enhanced piezoelectric properties to the III-V NWs [21] (discussed below in detail), this property has been virtually overlooked experimentally, compared to the research efforts invested in studying the electronic and optical properties of III-V NWs. We are currently aware of only two reports regarding the piezoelectric activity of WZ GaAs NW ensembles, yielding a possible range for the value of the $d_{33}$ coefficient $[22,23]$. In a different study, piezoelectricity in a WZ InAs NW is related to changes in a two terminal device conductance [24], through the piezotronic effect [8]. Developing a fundamental understanding of the piezoelectric properties of these NWs is crucial for piezo-phototronic applications in the visible range, sensing, as well as energy harvesting. The possible benefits are significant considering the wide knowledge base available for these materials, and the possible integration with silicon based technology [10,25].

Herein, we explore the piezoelectricity of single III-V NWs, with ZB and WZ structures. We consider the piezoelectric characteristics expected for [111] oriented NWs, showing finite element (FE) simulations of piezo-response force microscopy (PFM) on such NWs, and present PFM measurements on horizontal and vertical NWs, in light of simulation results. PFM is the leading method for carrying-out direct piezoelectric characterization [26-28]; nonetheless, due to it being a contactmode atomic force microscopy (AFM) method, its application to samples other than thin-films is particularly challenging. We therefore apply a novel non-destructive PFM measurement method suitable for studying NWs, which we briefly discuss here, and details of which will be published elsewhere. It should be noted that while PFM measurements have previously been conducted on III-N NWs [29], such measurements have not been attempted before in III-V NW, to the best of our knowledge. Our work therefore, presents the first direct PFM studies on III-V NWs, which is validated through computational modelling. 


\section{Scientific Background}

\subsection{Piezoelectricity in III-V NWs}

The piezoelectric properties of bulk III-V have been known for decades, and have been successfully used to realize electromechanical applications [30,31]. In the ZB structure, when the principal axes of the piezoelectric matrix are aligned with the cubic axes, III-V materials exhibit only shear piezoelectricity, $d_{14} \neq 0$. The piezoelectric matrix takes the form of:

$$
d_{Z B,[001]}=\left(\begin{array}{cccccc}
0 & 0 & 0 & d_{14} & 0 & 0 \\
0 & 0 & 0 & 0 & d_{14} & 0 \\
0 & 0 & 0 & 0 & 0 & d_{14}
\end{array}\right)
$$

where for GaAs, $d_{Z B, 14, G a A s}=2.6 \mathrm{pm} / \mathrm{V}$, and for $\operatorname{InP} d_{Z B, 14, G a A s}=1.8$.

For III-V $N W s$, the picture is more complex: firstly, as mentioned earlier, NWs grow both in ZB and WZ structure, having distinct piezoelectric coefficients; secondly, the prevailing growth direction is [111]/[0001] (in ZB/WZ notations) [15]. For WZ NWs, this growth direction aligns with the polarized axis (3-axis of the piezoelectric matrix), therefore no matrix adjustments are needed for considering WZ NW piezoelectricity. However, in ZB structure, for practical reasons (there is negligible spontaneous polarization in ZB structures [4] so the polarized axis is chosen arbitrarily) the matrix should be rotated such that 3-axis corresponds to [111], with the following rotation matrix [32]:

$$
a=\left(\begin{array}{ccc}
\frac{1}{\sqrt{6}} & \frac{1}{\sqrt{6}} & -\sqrt{\frac{2}{3}} \\
-\frac{1}{\sqrt{2}} & \frac{1}{\sqrt{2}} & 0 \\
\frac{1}{\sqrt{3}} & \frac{1}{\sqrt{3}} & \frac{1}{\sqrt{3}}
\end{array}\right) .
$$

The piezoelectric matrices for WZ and ZB in [111]/[0001] NW coordinates become [32]:

$$
\begin{aligned}
d_{W Z,[0001]} & =\left(\begin{array}{cccccc}
0 & 0 & 0 & 0 & d_{15} & 0 \\
0 & 0 & 0 & d_{15} & 0 & 0 \\
d_{31} & d_{31} & d_{33} & 0 & 0 & 0
\end{array}\right) \\
d_{Z B,[111]} & =\left(\begin{array}{cccccc}
d_{11} & -d_{11} & 0 & 0 & d_{15} & 0 \\
0 & 0 & 0 & d_{15} & 0 & d_{26} \\
d_{31} & d_{31} & d_{33} & 0 & 0 & 0
\end{array}\right)
\end{aligned}
$$

with the 1 -axis corresponding to $[11 \overline{2}] /[10 \overline{1} 0]$ and the 2 -axis corresponds to $[\overline{1} 10] /[\overline{1} 2 \overline{1} 0]$. The numerical values are

$$
d_{G a A s, Z B,[111]}=\left(\begin{array}{cccccc}
-1.06 & 1.06 & 0 & 0 & -1.50 & 0 \\
0 & 0 & 0 & -1.50 & 0 & 2.12 \\
-0.75 & -0.75 & 1.50 & 0 & 0 & 0
\end{array}\right) \mathrm{pm} / \mathrm{V}
$$


There are no experimental coefficients for single WZ III-V NWs (Lysak et al. reported on NW ensemble [23]), and so the values we use are theoretical $[21,33,34]$

$$
d_{\text {GaAs,WZ,[0001] }}=\left(\begin{array}{cccccc}
0 & 0 & 0 & 0 & d_{15} & 0 \\
0 & 0 & 0 & d_{15} & 0 & 0 \\
-1.15 & -1.15 & 2.3 & 0 & 0 & 0
\end{array}\right) \mathrm{pm} / \mathrm{V}
$$

A similar procedure for InP in the WZ form yields

$$
d_{I n P, W Z,[0001]}=\left(\begin{array}{cccccc}
0 & 0 & 0 & 0 & d_{15} & 0 \\
0 & 0 & 0 & d_{15} & 0 & 0 \\
-2.46 & -2.46 & 5.42 & 0 & 0 & 0
\end{array}\right) \mathrm{pm} / \mathrm{V}
$$

Since Al-zahrani et al. do not report a theoretical value for the piezoelectric constant $e_{15}$ [21], we do not report a $d_{15}$ value; nonetheless, for simulation purposes we have used $d_{G a A s, W Z, 15}=-3.3 \mathrm{pm} / \mathrm{V}$, following Lysak and co-workers [23]. It is noteworthy that in the [111] orientation, ZB NWs are expected to have an axial $\left(d_{33}\right)$ response, and that additional coefficients are expected to come into play, when compared to WZ NWs (single GaN NWs were recently examined [29], in order to extract the three WZ coefficients). Furthermore, when comparing similar coefficients, the WZ structure is expected to yield a stronger piezoelectric response. In the following sections we examine these effects in detail.

Figure $2 \mathrm{~b}$ shows a schematic representation of $\mathrm{ZB}$ and $\mathrm{WZ}$ structures. Although plotted ideally, the WZ structure induces non-idealities in the anion and cation location resulting in spontaneous polarisation, exploited in in III-N based electronic devices [4]. Interestingly, for GaN NWs, a large value, $d_{33, G a N, N W}=12-13 \mathrm{pm} / \mathrm{V}$, has been reported [29], (compared to bulk values $d_{33, G a N}=3-4$ ) indicating that NW geometry is preferable for piezoelectric applications. Due to their associated electronic and optical properties relevant for piezotronic and piezo-phototronic applications respectively, III-V NWs make a particularly interesting piezoelectric research subject.

\section{Experimental}

\subsection{Nanowire growth and sample preparation}

Self-catalyzed GaAs NWs were grown on epiready n-doped Si substrates with (111) orientation by solid-source molecular beam epitaxy (MBE). Before introduction into the ultra-high vacuum (UHV) system, the substrate was ultrasonically cleaned by immersion into acetone ( $5 \mathrm{~min}$ ) and then ethanol $(5 \mathrm{~min})$, in order to remove surface contamination. The rinsed substrate was then out-gassed at $200^{\circ} \mathrm{C}$ under UHV for a few minutes and then transferred into the III-V dedicated MBE chamber. During growth, the sample holder was continuously rotated to enhance the growth homogeneity. Vapour-liquidsolid (VLS) mechanism is used for the growth of GaAs NW array, with Ga droplets 
serving as catalysts. The temperature of the Si substrate, whose native oxide was still on the surface, was first increased to $530^{\circ} \mathrm{C}$ and 1 nominal monolayer (ML) of Ga was deposited on the substrate at an equivalent GaAs 2D growth rate of $0.50 \mathrm{ML} / \mathrm{s}$. The Ga atoms lead to the local decomposition of the native oxide on the Si surface [35] creating pinholes and defective sites [36]. This effect enhances the accumulation of Ga atoms to form Ga droplets for the following NW growth. Then substrate temperature was increased to $610^{\circ} \mathrm{C}$ for the axial growth of NWs, initiated by the Ga and As flux. The GaAs growth rate was set at about $0.75 \mathrm{ML} / \mathrm{s}$, with a beam equivalent pressure (BEP) of 3.6 $\cdot 10^{-7}$ Torr and $3.6 \cdot 10^{-6}$ Torr for Ga the $\mathrm{As}_{4}$ respectively. After 20 min of deposition, the Ga flux was cut off and the sample was cooled down to room temperature (RT). As GaAs inclines to decompose at high temperature, an $\mathrm{As}_{4}$ atmosphere was maintained during the cooling procedure.

Figure 1a shows an as-prepared GaAs NW array on Si substrate with a density
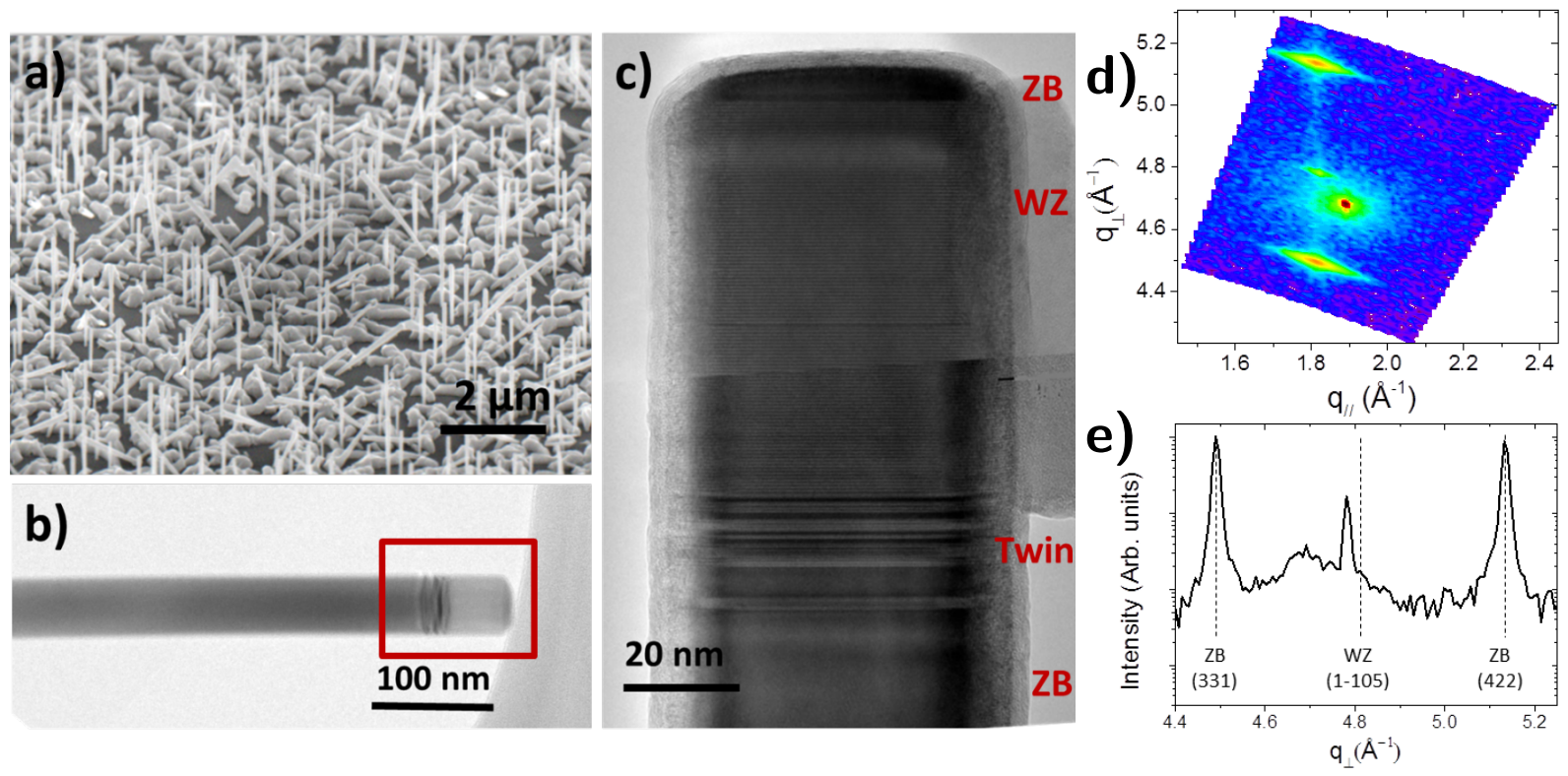

Figure 1: Nanowire characterization: a) SEM image of GaAs NW array on n-Si(111); b) TEM image of one typical GaAs NW; c) HRTEM image of the tip of one typical GaAs NW; d) X-ray diffraction reciprocal space map; e) Diffracted intensity plotted along the out of plane scattering vector. The diffuse scattering of the Si substrate is visible close to the WZ [1]̄05] peak.

of $5.5 \mathrm{NW} / \mu \mathrm{m}^{2}$. A typical $\mathrm{NW}$ is about $2 \mu \mathrm{m}$ with an average diameter about $80 \mathrm{~nm}$ (Figure 1a,b). Most of the NWs are vertical, epitaxially grown on the Si substrate. Figure 1c shows the high resolution TEM (HRTEM) image of the tip of a typical GaAs NW (marked area in Figure 1b), clearly revealing the four different parts of the NW: the first segment is a perfectly pure ZB region (starting from the substrate) followed by the second segment, a transition region with high density of twin boundaries and stacking faults; the third one is a defect-free wurtzite (WZ) phase and finally the NW ends to an ultra-thin ZB part about $15 \mathrm{~nm}$. There is a thin layer of amorphous oxide 
on the surface of the NW because of oxidation in air (Figure 1c). Figure 1d shows the reciprocal space mapping performed on the epitaxial GaAs NWs. Measurements were performed with a laboratory diffractometer equipped with a rotating anode, the X-ray beam was monochromatized at $\lambda=1.5406 \AA$. By modifying the incidence angle and the detection angle at a fixed azimuthal angle, the reciprocal space was mapped without moving the sample, allowing the observation of $\{422\} \mathrm{ZB},\{331\} \mathrm{ZB}$ and $\{1 \overline{1} 05\} \mathrm{WZ}$ Bragg spots. As shown in Ref. 37 this mapping allows for a clear observation of the ZB and WZ parts of epitaxial GaAs NWs. Despite the proximity of a Si substrate Bragg spot close to the [1105] WZ reflection, the contribution of WZ crystal phase is clearly evidenced.

The map of Figure 1d allows to extract the in-plane and out-of-plane lattice parameters of both $\mathrm{ZB}$ and $\mathrm{WZ}$ phases. The measured lattice parameters are in agreement with Ref. 37 showing the presence of a bulk-like ZB structure (fully relaxed) while the WZ phase exhibits a modification of the out-of-plane lattice parameter (c $=6.5696 \AA$ ) corresponding to a c/a ratio of 1.645 , in good agreement with previous literature [37-39]. Finally, from the intensity of WZ and ZB Bragg peaks, the relative amount of $\mathrm{WZ}$ phase is about $10 \%$ of the crystalline material. This indicates a fraction which is slightly larger than that observed in the TEM images of nominally identical NWs, where the WZ segment is roughly $100 \mathrm{~nm}$ in 2 micron long NWs.

Similar crystal phase sequences (ZB, stacking faults, WZ and ZB) have been reported by several groups [36,40-43]. It is well established that GaAs crystal phase is depending on the contact angle of the droplet catalyst with the solid phase which can be tuned by the V/III ratio for instance [40]. In our experiment the growth was achieved at rather low $\mathrm{V} / \mathrm{III}$ ratio which induces the formation of $\mathrm{ZB}$ phase because the triple line is located on the $\{1 \overline{1} 0\}$ facets of the NWs (Figure 2a). However, the catalyst can be consumed during the growth which induces a modification of the contact angle. When the volume of the droplet is too small, the triple line is located on the top of the growing facets which favor the formation of the WZ phase (Figure 2a). The defective region made of $\mathrm{ZB}$ structure with stacking faults and twin boundaries is the consequence of the transition between the two regimes. The last part of the NWs is made of a small ZB area in agreement with the final consumption of the Ga droplet. The NWs were transferred to a gold-coated silicon substrate for PFM measurements by mechanical scraping of the substrates.

InP NWs were grown by metal organic molecular beam epitaxy (MOMBE), utilizing the selective-area vapor liquid solid method (SAVLS), details of which have been extensively discussed elsewhere [16,44]. Briefly, an n-type InP (111)B substrate was coated by plasma enhanced chemical vapour deposition (PECVD) silicon nitride, and subsequently patterned by e-beam lithography to etch pinholes (of about $40 \mathrm{~nm}$ ) in the selective area mask, where gold (4.5 nm thick) was deposited. Upon heating inside the growth chamber the gold became a liquid growth catalyst. The conditions used here were shown to result in the growth of pure WZ NWs [16]. Nanowire morphology and AFM images are shown later in Fig. 5. The NW height was found to be $\sim 1 \mu \mathrm{m}$, with 
(a)

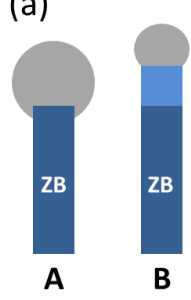


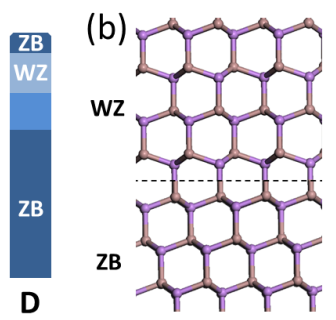

Figure 2: a) Growth mechanism of the ZB/WZ GaAs NWs; b) model of a ZB/WZ interface viewed down the $[\overline{1} 10]$ orientation.

diameter of $\sim 60 \mathrm{~nm}$. The sample was mounted on a conductive AFM setup as-grown, i.e. with the NWs vertically aligned. The NWs reported here are $300 \mathrm{~nm}$ stems of NWs left following damage to the $1 \mu \mathrm{m}$ NWs.

\subsection{Finite element analysis}

COMSOL Multiphysics 5.2a was used to model the PFM response of an individual GaAs NW, with the same morphology as described above, using Finite Element Analysis (FEA). A GaAs NW with a hexagonal cross section was placed horizontally on a rigid substrate which was set at a potential of $4 \mathrm{~V}$. An electrically grounded PFM tip was scanned either along the length of the NW, in contact with the uppermost surface, or across the NW (Figure S1 in Supporting Information). The total deformation of the nanowire was calculated for each tip position. From each of these solutions, the $x, y$ and $z$ deformation components of the point on the nanowire directly beneath the tip were extracted and used to build the simulated vertical and lateral PFM signals. Two orthogonal lateral (in-plane) signals were calculated, axial: displacement of the wire along its length, and transverse: in plane displacement of the nanowire perpendicular to its length. In the results, these two lateral signals are plotted together, although experimentally only one of these signals will be observed, dependent on the orientation of the tip cantilever with respect to the nanowire.

For the simulated scans along the NW, 3 segments were considered. The first and last segments consisted of ZB GaAs, oriented such that the [111] direction was parallel to the $z$ direction. The middle $50 \mathrm{~nm}$ segment consisted of WZ GaAs, with [0001] parallel to the $z$ axis. The crystallographic orientation of ZB GaAs was achieved by assigning the material a local coordinate system, rotated from the global $x y z$ axes using the matrix

$$
a^{-1}=\left(\begin{array}{ccc}
\frac{1}{\sqrt{6}} & \frac{-1}{\sqrt{2}} & \frac{1}{\sqrt{3}} \\
\frac{1}{\sqrt{6}} & \frac{1}{\sqrt{2}} & \frac{1}{\sqrt{3}} \\
-\sqrt{\frac{2}{3}} & 0 & \frac{1}{\sqrt{3}}
\end{array}\right)
$$


This allowed for the piezoelectric and elastic properties to be entered as described in the material's principal axes. For scans across the NW, only ZB material was considered.

The boundaries between the segments ensured that the nanowire was mechanically continuous. The facet in contact with the substrate was set as a fixed boundary. The AFM tip apex was spherical with a radius of curvature of $50 \mathrm{~nm}$. The model calculated the steady-state response of the nanowire.

\subsection{PFM analysis}

Due to the nature of the sample at hand, including short horizontal NWs, and mechanical-exfoliation related debris (Fig. S2 in Supporting Information), or long vertical NWs (Fig. 5) traditional PFM application was not successful. The nondestructive PFM method adopted here for the study of NWs is described in length elsewhere. Briefly, the measurement is based on an intermittent AFM mode known as peak-force ${ }^{\mathrm{TM}}$ (Bruker), where the mechanical properties of the sample, as well as topography, are sensed by continuous acquisition of force-curves, and their on-line analysis [45]. By applying an off-resonance electrical voltage to the tip, and analysing the corresponding deflection only when the tip is in contact with the sample, the PFM signals (both vertical and lateral) are extracted (Figures S2-S3 in Supporting Information). Since this procedure relies upon a high speed data capture protocol (6.25 $\mathrm{MHz}$ sampling) to extract the tip deflection, only single line-scans are recorded at a time (Figure 4a). We have used a Nanoscope VIII (Bruker) in a modified peak-force tapping mode, with MESP-RC-V2 tips $(5-7 \mathrm{~N} / \mathrm{m}, \sim 150 \mathrm{kHz})$. The voltage amplitude applied to the substrate (with the tip being grounded) was $4-8 \mathrm{~V} @ 125 \mathrm{kHz}$. Note that the PFM frequency is much higher than the peak-force frequency used $(250 \mathrm{~Hz})$, such that there are several periods of PFM activation complete in the time the sip is in contact with the sample, which is about 0.5 msec.

\section{Results and discussion}

\subsection{Finite elements measurements}

Figure 3 schematically shows the simulated PFM configurations. In Fig. 3a,b the simulated NW was constructed to resemble the experimentally grown NWs: predominantly ZB, with a $50 \mathrm{~nm}$ WZ segment, and a ZB tip. The NW axis was aligned with the $[111] /[0001]$ orientation, therefore the piezoelectric matrices in Eq. 5 \& 6 can be used to interpret the results. Two configurations were examined - with the PFM tip applying a field down the 1-axis $([11 \overline{2}] /[10 \overline{1} 0])$ or alternatively down the 2-axis $([\overline{1} 10] /[\overline{1} 2 \overline{1} 0])$. In Fig. 3c the NW was treated as pure ZB and scanned perpendicular to its length. The crystalline configuration considered was identical to that Fig. 3b.

The simulated "vertical" and two orthogonal "lateral" PFM signals are also shown in Figure 3, corresponding to the schematic. It is evident that the ZB and WZ GaAs regions yield different PFM responses. Furthermore, the orientation of the nanowire on 
the substrate had a significant effect on the PFM response observed. Lateral signals were present in both orientations; in the $[11 \overline{2}]$ field orientation the lateral response was manifested only in the axial signal, whereas for a field applied along [110] a lateral response could be observed in both axial and transverse directions. Changing the nanowire orientation also led to a change in the vertical signal observed. Zinc blende GaAs yields a strong vertical response in the $[11 \overline{2}]$ field orientation, a result of the nonzero $d_{11, G a A s, Z B,[111]}$ coefficient in the rotated matrix in Eq. 5. Conversely, $d_{22, G a A s, Z B,[111]}$ in the same rotated matrix is zero, hence for the orthogonal field orientation [110], there was no vertical response from the $\mathrm{ZB}$ region. In each case, the transition from the $\mathrm{ZB}$ region to the $\mathrm{WZ}$ region resulted in different characteristics. Generally, in these configurations, the vertical WZ signal is expected to be low, considering the lack of a $d_{11} / d_{22}$ coefficient. Lateral signals are expected due to the $d_{15} / d_{24}$ shear modes.

To achieve the different field orientations in Figure $3 \mathrm{a} \& \mathrm{~b}$, the entire (simulated) nanowire was rotated $90^{\circ}$ about its axis, including the WZ region. From inspecting the piezoelectric matrix in Eq. 6, it is apparent that such a rotation should have no effect on the piezoelectric response of WZ GaAs with [0001] orientation. However, it is evident from Figure 3 that the PFM response from the WZ region does indeed change with nanowire orientation. This apparent discrepancy can be rationalised by considering that the WZ region is mechanically connected to the $\mathrm{ZB}$ region and that the influence of the electric field extends beyond the area immediately beneath the tip. Even as the tip was scanned along the WZ region, deformation was still induced within the ZB region. The deformation detected by the tip therefore had contributions from all elements both 'upstream' and 'downstream' of the tip position. Therefore, since the response from ZB GaAs changed with field orientation, the response observed from the WZ region also changed. As an example, consider the vertical signals from both field orientations. For a field along $[11 \overline{2}]$, ZB GaAs produced a large response, large enough to make the response from WZ GaAs undetectable. Only in the orthogonal field direction, where ZB GaAs is not active in the vertical direction, can the vertical response from WZ GaAs be observed. In both these cases, the vertical response from WZ GaAs was identical, yet the effect of the neighbouring ZB GaAs results in two different observed signals.

When the NW was scanned in a direction perpendicular to its length (Fig. 3c), features related to the geometry and tip position arose. For example, a stronger axial lateral signal was found when the tip contacted the side facets compared to the top facet. Conversely, the transverse lateral signal reached a maximum value on the top facet - a value which was larger than any other orientation or tip position. The vertical signal showed two peaks of opposite signs, and notably, the signal was not constant on the top facet, as was the case for the transverse lateral signal. Our experimental system is more likely to correspond to the two configurations on the right-hand side where field is applied along the 2-axis, considering: i) the side-facets of the NWs are $\{\overline{1} 10\}$; ii) it is more likely that a NW rests on its facet rather than its edge. This will be taken into account when examining experimental results. 
a
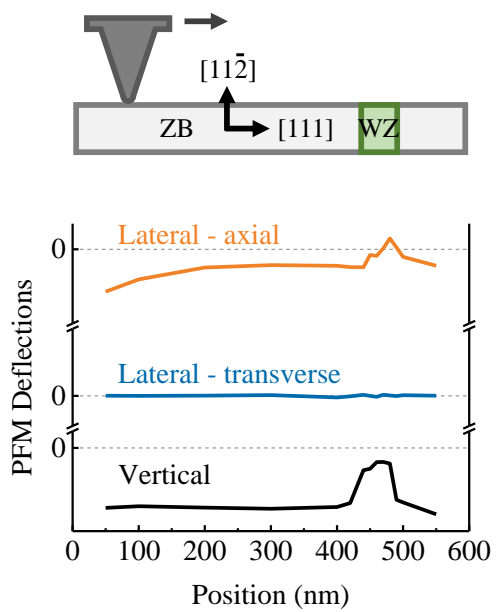

b
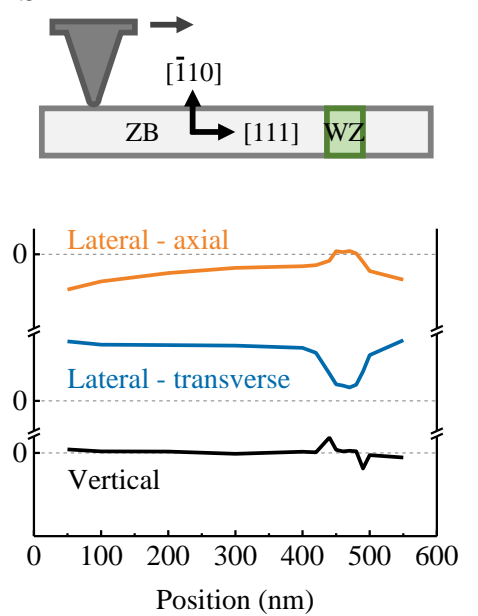

c
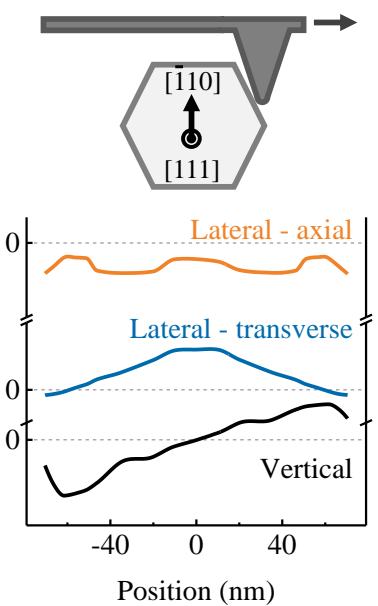

Figure 3: Simulated vertical and lateral PFM responses from horizontal GaAs nanowires in two different orientations scanned along their length, corresponding to a) a field along the piezoelectric ' 1 ' axis and b) ' 2 ' axis as described by the matrices in equation 4; c) a ZB NW scanned across its length, field applied at '2' axis. All signals are plotted in the same scale of arbitrary units. The tip in $[a, b]$ was modeled as a cone and sphere of $35 \mathrm{~nm}$ radius, while in [c] it was modeled as a sphere of $30 \mathrm{~nm}$ radius. The notations 'axial' and 'transverse' relate to the NW, not the cantilever.

\subsection{PFM measurements}

Figure 4a shows the peak-force based topography image of a NW ready for the intermittent PFM measurement. The dashed arrow indicates the PFM scan direction, and the thick arrow indicates the cantilever orientation, which determines if lateral-axial or lateral-transverse PFM signals are recorded. As mentioned above, it is reasonable to assume that the NWs were lying on a facet, and that the 2-axis of the piezoelectric coordinates is the vertical axis, and therefore the picture arising from Fig. 3c was expected. Figure 4b shows the extracted vertical and lateral PFM signals in arbitrary units, under an 8V PFM excitation; the NW topography (in arbitrary units) is shown as well. Considering the configuration of the cantilever and the NW, the lateral signal probed is the "lateral-axial" (top simulated curve) - dominated by $d_{24}$. The vertical signal measured in experiments was, unlike in the ideal simulation, intermixed with the transverse lateral signal, resulting in buckling mode deformations of the cantilever, added to the vertical deflection mode; the associated signal might actually be stronger than the "true" vertical signal [46].

Figure 4c,d show the vertical and lateral signals in the area marked by the dashed circle. Interestingly, features that can be related to the top and two side facets were seen, with transition taking place in similar positions in both channels, with reasonable fit to the simulation. Taking a closer look, the vertical channel could be thought of as a combination of the simulated vertical and transverse lateral signals, as mentioned above. 
This is demonstrated by the dashed lines - resembling these features in the simulation. The lateral signal also bore resemblance to the middle section of the simulated axiallateral signal. Following a calibration procedure (Figure S4 in Supporting Information) the vertical measurement sensitivity was $\operatorname{sim} 0.4 \mathrm{pm} / \mathrm{a} . \mathrm{u}$. (see Supporting Information), leading to coefficients of the order of $0.5 \mathrm{pm} / \mathrm{V}$. Note that in this measurement, a kelvin probe force microscopy (KPFM) measurement was performed prior to the PFM, and the measured contact potential was used applied in order to reduce electrostatic contribution to the signal [47]. When considering other factors affecting the measurement, it is possible that surface states and oxide layer on the GaAs surface were interfering with better assessment of the coefficients. Furthermore, the complicated $d$-matrix of the ZB structure makes it even difficult to extract a quantitative coefficient. Nonetheless, the value reached is reasonably close to the theoretical coefficients of GaAs, in various orientations.

Figure 5a,b show the topography of an InP NW array obtained by SEM and
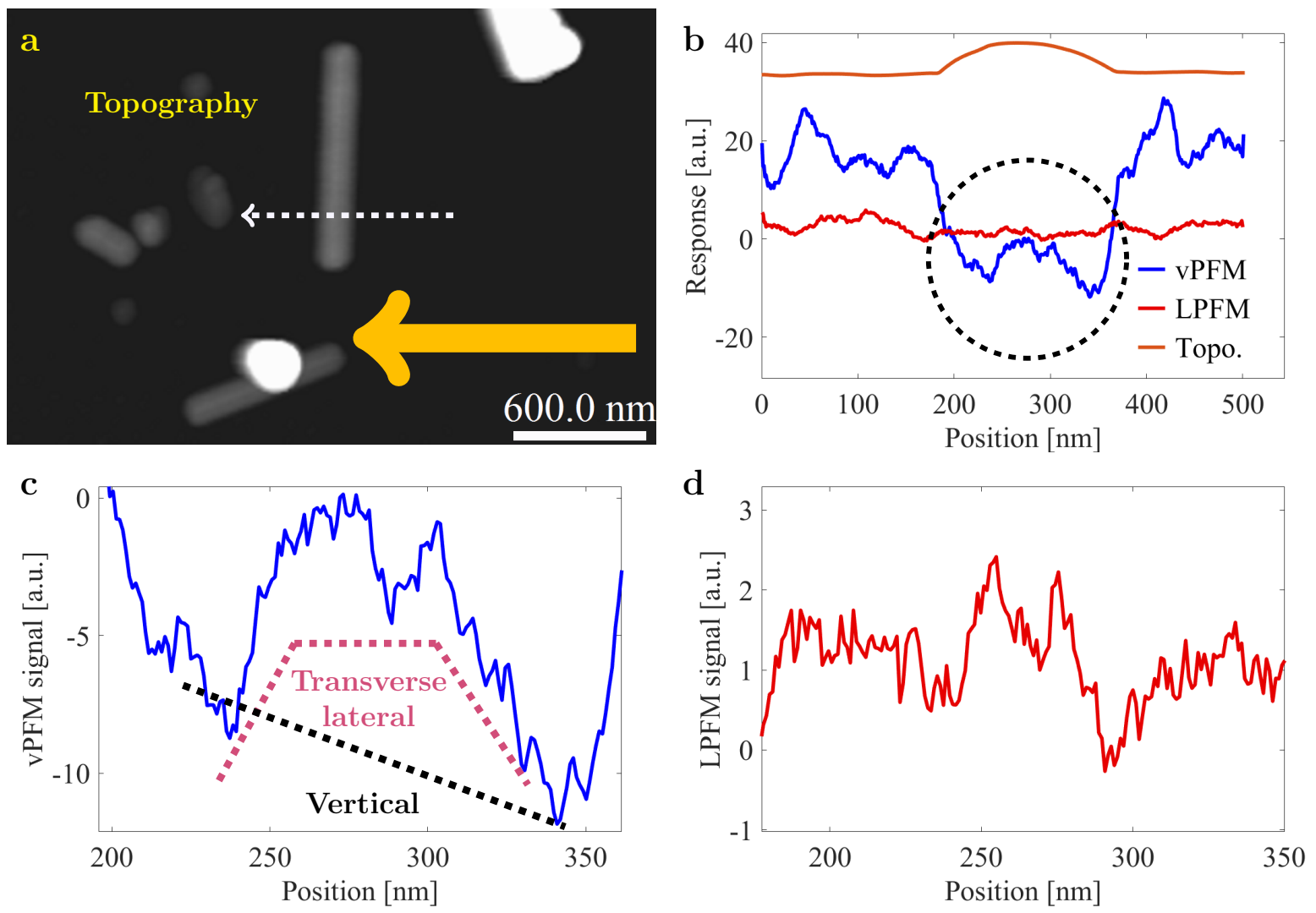

Figure 4: Peak-force PFM measurements of a GaAs NW lying transverse to the cantilever. a) NW topography, with scan direction and cantilever orientation (dashed and solid arrows correspondingly); b) uncalibrated PFM results, showing the vertical and lateral signals, alongside the scan topography. The dashed circle indicates the area of interest; c) close-up to the vertical PFM signal; d) close-up to the lateral PFM signal.

tapping mode AFM (prior to peak-force mode onset), correspondingly. The inset shows 
the peak-force image of two NWs which broke under the AFM tip during attempts to perform the PFM measurements. It is likely that these NWs, of about $1 \mu \mathrm{m}$ in length, were too long for stable peak-force operation. However, the remaining stem of about $300 \mathrm{~nm}$ was the subject of a PFM scan, shortly after its failure. Figure 5c shows the vertical PFM signal extracted from the measurement, alongside the topography, under a 4V PFM excitation. Figure 5d shows a close-up to the signal containing both substrate and NW. The selective area mask is expected to impede most of the PFM signal, and is treated as zero (the offset attributed to background noise in PFM [48]), resulting in a signal of about 7 a.u. from the NW, corresponding to about $3 \mathrm{pm}$ displacement, considering the calibration described earlier. This leads to a $d_{33}$ coefficient of about 0.7-1 pm/V for the vertical InP NW. This value is somewhat smaller than expected however is the first report of the piezoelectric coefficient of a non-nitride III-V NW. We note that the $d_{31}$ coefficient might contribute to this signal through the buckling mode of the cantilever, and therefore future work should include examining vertical NW by scanning in orthogonal directions. We note that the lateral signal is this case did not exhibit distinctive results when comparing the NW and the substrate.

PFM scans taken along the NW axis were inconclusive in clearly distinguishing the ZB and WZ segments, and are currently under investigation. This is most likely due to tip being of comparable size with the WZ segment, and the presence of surface states. The use of NWs with longer segment of different crystalline structures might be beneficial to improve detection of such signals. However, the simulations shown here shed light on the complexity of the topic and serve to inspire future work on the subject.

\section{Conclusions}

To conclude, in this work we examine in detail piezoelectricity in III-V NWs in light of recent interest in their piezotronic and piezo-phototronic applications. Such studies have not been reported before, due to challenges related to conventional contact mode PFM studies of nanomaterials. Our studies on III-V NWs are particularly significant when compared to III-V bulk materials as: i) WZ crystal structure is frequently observed in these NWs, and is expected to induce enhanced piezoelectric properties; ii) since the preferred growth direction of III-V NWs is [111], axial piezoelectricity is expected even in ZB structured NWs. We have further simulated the PFM response of horizontal GaAs NWs, illustrating the effect of tip/NW interaction, tip/NW positioning, scan direction, NW crystal structure and dimensionality. These simulations were directly used to discuss PFM measurements that have been performed for the first time on individual III-V NWs. In particular, we present results originating from a novel nondestructive PFM mode that is being specifically developed to study such NWs. In case of horizontal GaAs ZB NWs, our results were qualitatively explained by the simulation, and for the vertical WZ InP NW measured, an axial $d_{33}$ coefficient of about 0.7-1 $\mathrm{pm} / \mathrm{V}$ was extracted for the first time, reasonably in line with theoretical expectations. We hope our simulations and experiments will serve to inspire further work in this 

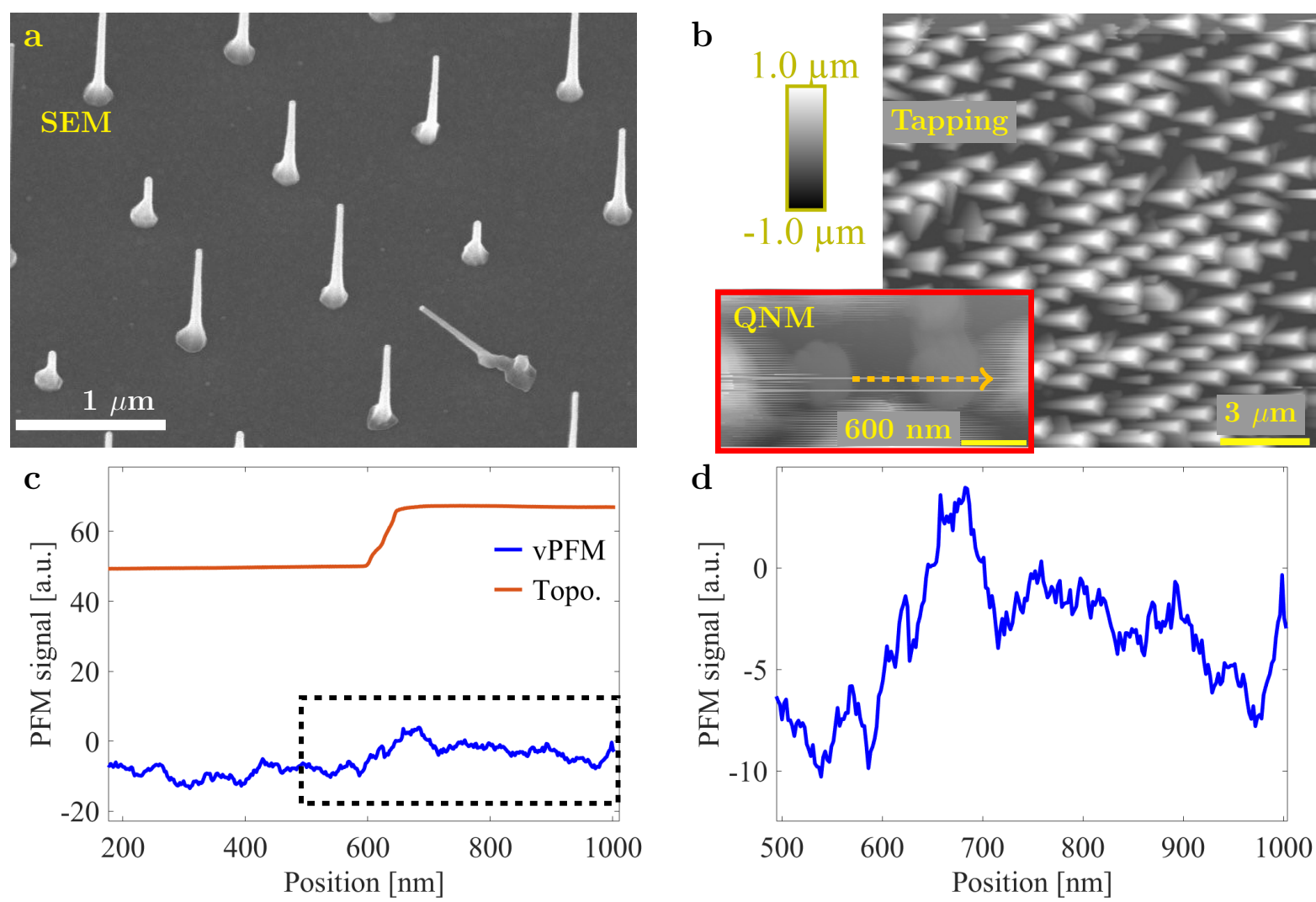

Figure 5: Peak-force PFM measurements of a vertical InP NW. a) Tilted SEM image of the examined array; b) NW array topography - obtained by tapping mode prior to QNM imaging. Inset shows QNM topography of two NW broken under the AFM tip - becoming a source for a PFM signal, the dashed arrow indicates the PFM scan; c) Uncalibrated PFM results, showing the vertical signal, alongside the scan topography. The dashed rectangle indicates the area of interest; d) close-up to the PFM signal.

fascinating aspect of III-V NWs.

\section{Acknowledgments}

S.K.-N. and Y.C. are grateful for financial support from the European Research Council through an ERC Starting Grant (Grant No. ERC-2014-STG-639526, NANOGEN). M.S is acknowledge studentship funding from the Cambridge Commonwealth, European \& International Trust. M. Gendry and J. Becdelievre are acknowledged for fruitful discussions and help with the sample preparation.

\section{References}

[1] Zhong Lin Wang and Jinhui Song. Piezoelectric nanogenerators based on zinc oxide nanowire arrays. Science, 312(5771):242-246, 2006.

[2] Xudong Wang, Jun Zhou, Jinhui Song, Jin Liu, Ningsheng Xu, and Zhong L Wang. Piezoelectric 
field effect transistor and nanoforce sensor based on a single $\mathrm{ZnO}$ nanowire. Nano letters, 6(12):2768-2772, 2006.

[3] N Gogneau, N Jamond, P Chrtien, F Houz, E Lefeuvre, and M Tchernycheva. From single III-nitride nanowires to piezoelectric generators: New route for powering nomad electronics. Semiconductor Science and Technology, 31(10):103002, 2016.

[4] Colin Wood and Debdeep Jena. Polarization effects in semiconductors: from ab initio theory to device applications. Springer Science \& Business Media, 2007.

[5] Ravi Agrawal and Horacio D Espinosa. Giant piezoelectric size effects in zinc oxide and gallium nitride nanowires. A first principles investigation. Nano letters, 11(2):786-790, 2011.

[6] Hemtej Gullapalli, Venkata SM Vemuru, Ashavani Kumar, Andres Botello-Mendez, Robert Vajtai, Mauricio Terrones, Satish Nagarajaiah, and Pulickel M Ajayan. Flexible piezoelectric ZnOpaper nanocomposite strain sensor. small, 6(15):1641-1646, 2010.

[7] Noelle Gogneau, Pascal Chrétien, Elisabeth Galopin, Stephane Guilet, Laurent Travers, JeanChristophe Harmand, and Frédéric Houzé. Gan nanowires for piezoelectric generators. physica status solidi (RRL)-Rapid Research Letters, 8(5):414-419, 2014.

[8] Zhong Lin Wang. Piezopotential gated nanowire devices: Piezotronics and piezo-phototronics. Nano Today, 5(6):540-552, 2010.

[9] Qing Yang, Xin Guo, Wenhui Wang, Yan Zhang, Sheng Xu, Der Hsien Lien, and Zhong Lin Wang. Enhancing sensitivity of a single $\mathrm{ZnO}$ micro-/nanowire photodetector by piezo-phototronic effect. Acs Nano, 4(10):6285-6291, 2010.

[10] Katsuhiro Tomioka, Masatoshi Yoshimura, and Takashi Fukui. A III-V nanowire channel on silicon for high-performance vertical transistors. Nature, 488(7410):189-192, 2012.

[11] Aniruddha Konar, John Mathew, Kaushik Nayak, Mohit Bajaj, Rajan K Pandey, Sajal Dhara, KVRM Murali, and Mandar M Deshmukh. Carrier Transport in High Mobility InAs Nanowire Junctionless Transistors. Nano letters, 15(3):1684-1690, 2015.

[12] Peter Krogstrup, Henrik Ingerslev Jørgensen, Martin Heiss, Olivier Demichel, Jeppe V Holm, Martin Aagesen, Jesper Nygard, and Anna Fontcuberta i Morral. Single-nanowire solar cells beyond the Shockley-Queisser limit. Nature Photonics, 2013.

[13] Ziyuan Li, Xiaoming Yuan, Lan Fu, Kun Peng, Fan Wang, Xiao Fu, Philippe Caroff, Thomas P White, Hark Hoe Tan, and Chennupati Jagadish. Room temperature GaAsSb single nanowire infrared photodetectors. Nanotechnology, 26(44):445202, 2015.

[14] Yusuke Kitauchi, Yasunori Kobayashi, Katsuhiro Tomioka, Shinjiro Hara, Kenji Hiruma, Takashi Fukui, and Junichi Motohisa. Structural Transition in Indium Phosphide Nanowires. Nano Lett., 10(5):1699-1703, 2010.

[15] Sebastian Lehmann, Jesper Wallentin, Daniel Jacobsson, Knut Deppert, and Kimberly A Dick. A general approach for sharp crystal phase switching in InAs, GaAs, InP, and GaP nanowires using only group V flow. Nano letters, 13(9):4099-4105, 2013.

[16] A Kelrich, VG Dubrovskii, Y Calahorra, S Cohen, and D Ritter. Control of morphology and crystal purity of InP nanowires by variation of phosphine flux during selective area MOMBE. Nanotechnology, 26(8):085303, 2015.

[17] Simon M Sze and Kwok K Ng. Physics of semiconductor devices. John wiley \& sons, 2006.

[18] Frank Glas, Jean-Christophe Harmand, and Gilles Patriarche. Why does wurtzite form in nanowires of III-V zinc blende semiconductors? Physical review letters, 99(14):146101, 2007.

[19] Hannah J Joyce, Jennifer Wong-Leung, Qiang Gao, H Hoe Tan, and Chennupati Jagadish. Phase perfection in zinc blende and wurtzite III- V nanowires using basic growth parameters. Nano letters, 10(3):908-915, 2010.

[20] Masanari Koguchi, Hiroshi Kakibayashi, Masamitsu Yazawa, Kenji Hiruma, and Toshio Katsuyama. Crystal structure change of GaAs and InAs whiskers from zinc-blende to wurtzite type. Japanese journal of applied physics, 31(7R):2061, 1992.

[21] Hanan YS Al-Zahrani, Joydeep Pal, Max A Migliorato, Geoffrey Tse, and Dapeng Yu. Piezoelectric field enhancement in III-V core-shell nanowires. Nano Energy, 14:382-391, 2015. 
[22] IP Soshnikov, Dm E Afanasev, VA Petrov, GE Cirlin, AD Bouravlev, Yu B Samsonenko, A Khrebtov, EM Tanklevskaya, and IA Seleznev. Piezoelectric effect in GaAs nanowires. Semiconductors, 45(8):1082-1084, 2011.

[23] Volodymyr V Lysak, Ilya P Soshnikov, Erkki Lahderanta, and George E Cirlin. Piezoelectric effect in wurtzite GaAs nanowires: Growth, characterization, and electromechanical 3D modeling. physica status solidi (a), 213(11):3014-3019, 2016.

[24] Xing Li, Xianlong Wei, Tingting Xu, Dong Pan, Jianhua Zhao, and Qing Chen. Remarkable and Crystal-Structure-Dependent Piezoelectric and Piezoresistive Effects of InAs Nanowires. Advanced Materials, 27(18):2852-2858, 2015.

[25] Eleonora Russo-Averchi, Martin Heiss, Lionel Michelet, Peter Krogstrup, Jesper Nygard, Cesar Magen, Joan Ramon Morante, Emanuele Uccelli, Jordi Arbiol, and A Fontcuberta i Morral. Suppression of three dimensional twinning for a $100 \%$ yield of vertical GaAs nanowires on silicon. Nanoscale, 4(5):1486-1490, 2012.

[26] A Gruverman and Sergei V Kalinin. Piezoresponse force microscopy and recent advances in nanoscale studies of ferroelectrics. Journal of materials science, 41(1):107-116, 2006.

[27] Yonatan Calahorra, Richard A Whiter, Qingshen Jing, Vijay Narayan, and Sohini Kar-Narayan. Localized electromechanical interactions in ferroelectric P (VDF-TrFE) nanowires investigated by scanning probe microscopy. APL Materials, 4(11):116106, 2016.

[28] Michael Smith, Yonatan Calahorra, Qingshen Jing, , and Sohini Kar-Narayan. Direct Observation of Shear Piezoelectricity in Poly-L-lactic Acid Nanowires. APL Materials, In press.

[29] Majid Minary-Jolandan, Rodrigo A Bernal, Irma Kuljanishvili, Victor Parpoil, and Horacio D Espinosa. Individual gan nanowires exhibit strong piezoelectricity in 3d. Nano letters, 12(2):970-976, 2012.

[30] Klas Hjort, Jan Soderkvist, and J-A Schweitz. Gallium arsenide as a mechanical material. Journal of Micromechanics and Microengineering, 4(1):1, 1994.

[31] Fabio Bernardini, Vincenzo Fiorentini, and David Vanderbilt. Spontaneous polarization and piezoelectric constants of III-V nitrides. Physical Review B, 56(16):R10024, 1997.

[32] Seoung-Hwan Park and Shun-Lien Chuang. Comparison of zinc-blende and wurtzite GaN semiconductors with spontaneous polarization and piezoelectric field effects. Journal of Applied Physics, 87(1):353-364, 2000.

[33] SQ Wang and HQ Ye. First-principles study on elastic properties and phase stability of III-V compounds. physica status solidi (b), 240(1):45-54, 2003.

[34] T Hanada. Basic properties of $\mathrm{ZnO}, \mathrm{GaN}$, and related materials. In Oxide and Nitride Semiconductors, pages 1-19. Springer, 2009.

[35] A Fontcuberta i Morral, Carlo Colombo, Gerhard Abstreiter, Jordi Arbiol, and JR Morante. Nucleation mechanism of gallium-assisted molecular beam epitaxy growth of gallium arsenide nanowires. Applied Physics Letters, 92(EPFL-ARTICLE-149095):063112, 2008.

[36] Young Heon Kim, Dong Woo Park, and Sang Jun Lee. Gallium-droplet behaviors of selfcatalyzed GaAs nanowires: A transmission electron microscopy study. Applied Physics Letters, 100(3):033117, 2012.

[37] Andreas Biermanns, Steffen Breuer, Anton Davydok, Lutz Geelhaar, and Ullrich Pietsch. Structural evolution of self-assisted GaAs nanowires grown on $\mathrm{Si}$ (111). physica status solidi (RRL)-Rapid Research Letters, 5(4):156-158, 2011.

[38] Chin-Yu Yeh, ZW Lu, S Froyen, and Alex Zunger. Zinc-blende-wurtzite polytypism in semiconductors. Physical Review B, 46(16):10086, 1992.

[39] Tawinan Cheiwchanchamnangij and Walter RL Lambrecht. Band structure parameters of wurtzite and zinc-blende GaAs under strain in the GW approximation. Physical Review B, 84(3):035203, 2011.

[40] Xuezhe Yu, Hailong Wang, Jun Lu, Jianhua Zhao, Jennifer Misuraca, Peng Xiong, and Stephan von Molnar. Evidence for structural phase transitions induced by the triple phase line shift in self-catalyzed GaAs nanowires. Nano letters, 12(10):5436-5442, 2012. 
[41] Torsten Rieger, Mihail Ion Lepsa, Thomas Schäpers, and Detlev Grützmacher. Controlled wurtzite inclusions in self-catalyzed zinc blende III-V semiconductor nanowires. Journal of crystal growth, 378:506-510, 2013.

[42] Peter Krogstrup, Ronit Popovitz-Biro, Erik Johnson, Morten Hannibal Madsen, Jesper Nygård, and Hadas Shtrikman. Structural phase control in self-catalyzed growth of gaas nanowires on silicon (111). Nano letters, 10(11):4475-4482, 2010.

[43] GE Cirlin, Vladimir G Dubrovskii, Yu B Samsonenko, AD Bouravleuv, K Durose, Yu Yu Proskuryakov, Budhikar Mendes, L Bowen, MA Kaliteevski, RA Abram, et al. Self-catalyzed, pure zincblende gaas nanowires grown on si (111) by molecular beam epitaxy. Physical Review $B, 82(3): 035302,2010$.

[44] A Kelrich, Y Calahorra, Y Greenberg, A Gavrilov, S Cohen, and D Ritter. Shadowing and mask opening effects during selective-area vapor-liquid-solid growth of InP nanowires by metalorganic molecular beam epitaxy. Nanotechnology, 24(47):475302, 2013.

[45] Bede Pittenger, Natalia Erina, and Chanmin Su. Quantitative Mechanical Property Mapping at the Nanoscale with PeakForce QNM. Bruker.

[46] Sergei V Kalinin, Brian J Rodriguez, Stephen Jesse, Junsoo Shin, Arthur P Baddorf, Pradyumna Gupta, Himanshu Jain, David B Williams, and Alexei Gruverman. Vector piezoresponse force microscopy. Microscopy and Microanalysis, 12(03):206-220, 2006.

[47] Sungho Kim, Daehee Seol, Xiaoli Lu, Marin Alexe, and Yunseok Kim. Electrostatic-free piezoresponse force microscopy. Scientific Reports, 7, 2017.

[48] T Jungk, A Hoffmann, and E Soergel. Consequences of the background in piezoresponse force microscopy on the imaging of ferroelectric domain structures. Journal of Microscopy, 227(1):72$78,2007$. 